\title{
Semiactive Nonsmooth Control for Building Structure with Deep Learning
}

\author{
Qing Wang, ${ }^{1}$ Jianhui Wang, ${ }^{1}$ Xiaofang Huang, ${ }^{2}$ and Li Zhang ${ }^{3}$ \\ ${ }^{1}$ School of Mechanical and Electric Engineering, Guangzhou University, Guangzhou 510006, China \\ ${ }^{2}$ Engineering Earthquake Resistance Center, Guangzhou University, Guangzhou 51045, China \\ ${ }^{3}$ Guangzhou Real Estate Management Vocational School, Guangzhou 510320, China
}

Correspondence should be addressed to Jianhui Wang; 87517619@qq.com

Received 13 July 2017; Revised 2 October 2017; Accepted 12 October 2017; Published 6 November 2017

Academic Editor: Guang Li

Copyright (C) 2017 Qing Wang et al. This is an open access article distributed under the Creative Commons Attribution License, which permits unrestricted use, distribution, and reproduction in any medium, provided the original work is properly cited.

\begin{abstract}
Aiming at suppressing harmful effect for building structure by surface motion, semiactive nonsmooth control algorithm with Deep Learning is proposed. By finite-time stable theory, the building structure closed-loop system's stability is discussed under the proposed control algorithm. It is found that the building structure closed-loop system is stable. Then the proposed control algorithm is applied on controlling the building structural vibration. The seismic action is chosen as El Centro seismic wave. Dynamic characteristics have comparative analysis between semiactive nonsmooth control and passive control in two simulation examples. They demonstrate that the designed control algorithm has great robustness and anti-interference. The proposed control algorithm is more effective than passive control in suppressing structural vibration.
\end{abstract}

\section{Introduction}

Seismic isolation technology for building structure has made great progress. And seismic isolation technology is used widely in many countries. However, seismic isolation system is along with large displacement when this system reduces seismic response of superstructure effectively. Thus, the application of seismic isolation technology is limited. In addition, the earthquake has strong randomness. If the designed passive isolation is used to suppress vibration, isolation parameters, which are used in earthquake action of some characteristics, not always have satisfying damping effect for other different characteristics earthquake. Hence, many scholars use control strategies to reduce displacement of isolating system. At the same time, the structural response is not added [1].

The study in regard to structural vibration control algorithm is a hot topic of structural vibration control field all the time [2]. In the last thirty years, structural vibration control technologies have made great progress. The technologies gradually become available means which ensure security of structure and resist the natural disasters. The so-called structural vibration control is used to change and adjust structural dynamic characteristic by deploying or inlaying some device or some equipment that applies force. And structural vibration control can obstruct and offset external excitation or dissipate energies that input to the structural system. The control actions can achieve the aim of controlling the structural dynamic characteristic reasonably. According to the characteristics of earthquake action, the structure that applied control measures possesses self-regulating ability of resisting earthquake action. Thereby, the structural intelligence comes true.

On the basis of having extra powers or not, the structural vibration control is divided into three classes [3]. The first class is passive control. The passive control does not need extra power. The structural vibration is suppressed only by interaction of control device and structure. Then the second class is active control that needs high-power extra power to drive actuator. The control is decided by measured excitation and feedback of structural response. The third class is semiactive control, which does not need to input great powers. Just a little power is applied when structural response is up to boundary value. And control device is switched to 
working state to reach parameters that adjust control system and the aim of reducing structural response.

The premise and foundation of structural vibration control are control algorithm. Therefore, according to features external disturbance, an important characteristic of structural vibration control is giving real control force by preset control algorithm. It guarantees that structural response is controlled to the safe range. And optimal control algorithm [4] gains lots of applications in civil structural control. However, the optimal control problem of forced vibration that earthquakes cause in long time does not establish extremum condition of optimal control. Thus, with the aid of COC (Classical Linear Optimal Control) [5], IOC (Instantaneous Optimal Control) [6], and LQG (Linear Quadratic Gaussian) control, the problem is improved. The other representative control algorithms [7-12] are LQR (Linear Quadratic Regulator) control, ILC (Iterative Learning Control), fuzzy logic control, and so on. Nevertheless, the aforementioned results still have large parameter perturbation of system and are oversensitive to external interference. It is extremely important to develop and apply the structural vibration control algorithm with stronger robustness. This kind of control algorithm has a little influence on the system parameters and has less sensitivity to external disturbances. After the 1990s, the American scholar Yang and others took the lead in the application of sliding mode control to vibration control of civil engineering structure. Sliding mode control, a switching control, makes the closed-loop system have the characteristics of finite-time convergence and strong robustness. But it is discontinuous for state variable of system. High-speed switching of control causes chattering phenomenon in the system. Nonsmooth control is able to achieve finite-time stability of system state. The robustness and inter-inference performance of system are both enhanced. Compared with traditional sliding mode control, nonsmooth control is continuous with respect to state variable. The control method receives attention and gains application [13-15] widely due to distinct advantages. Now nonsmooth control is studied and applied in the areas of design of high-precision guidance laws [16], attitude control of spacecraft [17], control of permanent magnet synchronous motor position [18], and so on.

However, nonsmooth control is not able to express uncertain information of system. Deep Learning is deep neural networks of a class of special nonclassified tags. Deep Learning usually has stackable denoising autoencoders and transformational autoencoders. The dimensions of their output vector and input vector are the same in some form of input vector generally. Expression of a data is studied or initial data is coded effectively by hidden layer. According to the study of literatures [19-29], Deep Learning can gain more representative characteristic information by training large scale data. Thus, the sample may be classified and estimated to improve precision of information.

In this paper, the nonlinear model of the building structure is described. According to expression of uncertain information with Deep Learning, the stability is deduced and analyzed in the based isolation structure by applying semiactive nonsmooth control algorithm. Under seismic wave called El Centro, the numerical simulation of two different examples is simulated numerically with semiactive nonsmooth control and the passive control. According to the comparison of simulation results, the feasibility and validity of the algorithm are verified.

\section{Mathematical Models and Related Theories}

2.1. Passive Isolation Structure. Some researchers, such as Zhou [4], Tang and Lium [30], and Kelly [31], do lots of work in seismic responses of passive isolation structure. It sets flexible seismic isolation between the bottom of superstructure and the top of foundation. Seismic isolation is used to prevent the superstructure from seismic energy [4]. The seismic isolation not only reduces seismic dynamic response of superstructure, but also ensures security of major structure and in-house facility effectively. In addition, the isolation system increases the natural vibration period. Due to increasing the natural period of the structure that exceeds the excitatory period of earthquake [4], isolation system avoids the phenomena of resonance and near resonance. Thus, isolation system reduces the seismic acceleration response of the structure.

The key to dynamic analysis of a building structure is the simplification of the structure. Two methods of structural simplification are described [32]. One is the way of lumpedmass; another one is the way of the centralized quality. If the distribution quality method is used to analyze dynamic, the structural equation of motion is a partial differential equation. It is not conducive for research of the practical application. Thus, the structural quality is often described by the lumped-mass method in practical engineering. It is simplified as a multi-degree-of-freedom story shear model. The superstructure and foundation of building are separated by seismic isolation. The energy, which is generated by seismic waves during the earthquake, is transferred to the seismic isolation from the base. And the seismic isolation prevents superstructure by self-deformation and vibration to dissipate energy [33].

In general, the horizontal stiffness of the seismic isolation bearing is much smaller than its vertical stiffness. While dynamic responses are analyzed, seismic isolation bearing is approximated to horizontal movement. And the vertical deformation that is caused by the swing is ignored. Compared with traditional structure, dynamic analysis of isolation structure consists of two parts. It consists of the isolation layer and the superstructure. In this paper, the building structure is simplified as one-dimensional story shear model. Under the action of horizontal earthquake, the equation of motion is represented by the following differential equation:

$$
M \ddot{X}+C \dot{X}+K X=-M R \ddot{x}_{g} .
$$

In this equation, $X=\left[\begin{array}{lllllll}x_{b} & x_{1} & x_{2} & \cdots & x_{i} & \cdots & x_{n}\end{array}\right]^{T}$ is displacement vector of the structure, where $x_{b}$ is base displacement relative to the ground and $x_{i}$ is displacement of the $i$ th floor relative to the ground. $\dot{X}$ and $\ddot{X}$ represent velocity vector and acceleration vector that include base velocity and acceleration. $\ddot{x}_{g}$ is a vector of ground acceleration. $R$ is a matrix of influence coefficient. $M, K$, and $C$ are mass matrix, 
proportional damping matrix, and stiffness matrix of the structure that include parameters of the isolation layer.

$$
\begin{aligned}
M & =\left[\begin{array}{cccc}
m_{b} & & & \\
& m_{1} & & \\
& & \ddots & \\
& & m_{n}
\end{array}\right] \\
K & =\left[\begin{array}{ccccc}
k_{b}+k_{1} & -k_{1} & & & \\
k_{1} & k_{1}+k_{2} & -k_{2} & & \\
& \ddots & \ddots & \ddots & \\
& & -k_{n-1} & k_{n-1}+k_{n} & -k_{n} \\
& & & -k_{n} & k_{n}
\end{array}\right] \\
C & =\left[\begin{array}{ccccc}
c_{b}+c_{1} & -c_{1} & & & \\
c_{1} & c_{1}+c_{2} & -c_{2} & & \\
& \ddots & \ddots & \ddots & \\
& & -c_{n-1} & c_{n-1}+c_{n} & -c_{n} \\
& & & -c_{n} & c_{n}
\end{array}\right] .
\end{aligned}
$$

2.2. Finite-Time Stability [14]. Semiactive nonsmooth control, which is introduced for the control system, can effectively improve the performance of the system. It realizes the finite-time stability of the system state and improves its robustness and anti-interference of the system. Combined with self-learning of the neural networks [34-37], note of hidden players is considered and the whole intelligence degree of the system is enhanced.

The finite-time stability problem of the following nonlinear systems is considered as

$$
\dot{z}=f(z), \quad f(0)=0, z \in R^{n}
$$

In this equation, a vector field of $C^{0}$ is $f(z)$.

Theorem 1. The homogeneous dilations of $z \in R^{n}$ are as follows:

$$
\Delta_{\varepsilon}^{\rho}(z)=\left[\varepsilon^{\rho_{1}} z_{1}, \varepsilon^{\rho_{2}} z_{2}, \ldots, \varepsilon^{\rho_{n}} z_{n}\right]^{T},
$$

where $\forall \varepsilon>0, \rho_{i}>0 i=1,2, \ldots, n$.

Theorem 2. For a vector field $f(z)$ in system (3), suppose there exists $l \in R$, and the function is satisfied as

$$
f_{i}\left(\Delta_{\varepsilon}^{\rho}(z)\right)=\varepsilon^{l+\rho_{i}} f_{i}(z) \quad i=1,2, \ldots, n .
$$

Then the vector field $f(z)$ is called vector fields homogeneous and related system is called homogeneous system. The homogeneous degree for the system is $l$.

Theorem 3. If system (3) is asymptotically stable and has convergence characteristic of finite-time, which means that the system state converges to equilibrium point of the system in finite-time, the system is finite-time stable. Equilibrium point 0 of system (3) has locally asymptotic stability. Equilibrium point 0 that is assumed is asymptotically global stability, while system (3) is a homogeneous system. And if the homogeneous degree in system is half negative $(l<0)$, system (3) is finite-time stable.

\section{Design of Semiactive Nonsmooth Control Algorithm with Deep Learning}

Passive control system can effectively reduce the loss caused by earthquakes, whereas the random of large earthquake is controlled difficultly. If seismic isolation produces the large displacement, its effect may be lost, which affects the whole performance of whole structure. In order to control the large displacement in the isolation layer, nonsmooth control algorithm is studied and designed. It can restrain the seismic wave that causes influence for building structural stability. In order to study the control algorithm conveniently, the maximum permissible displacement is assumed to be at the zero point. The related analysis is conducted.

3.1. Design of Nonsmooth Algorithm with Deep Learning. With the action of one-dimensional horizontal seismic acceleration, the equation of motion is as

$$
M \ddot{X}+C \dot{X}+K X=-M R \ddot{x}_{g}+E U .
$$

In this equation, $E$ is a position matrix of controllers. $U$ is a vector comprising control forces. The acceleration $\ddot{x}_{g}$ is unknown disturbance and established by Deep Learning neural network. In this paper, autoencoder neural network (see $[20,21]$ ) is used to establish $\ddot{x}_{g}$. Other parameters have been explained in (1).

Defining a state-space vector $Y=\left(\begin{array}{ll}Y_{1} & Y_{2}\end{array}\right)^{T}$, where $Y_{1}=$ $\left(\begin{array}{llll}Y_{11} & Y_{12} & \cdots & Y_{1 n}\end{array}\right)^{T}=X, Y_{2}=\left(\begin{array}{llll}Y_{21} & Y_{22} & \cdots & Y_{2 n}\end{array}\right)^{T}=\dot{X}$. The equation of motion is rewritten as $[6,38]$

$$
\begin{aligned}
\dot{Y} & =A Y+W \ddot{x}_{g}+B U \\
A & =\left[\begin{array}{cc}
0 & I_{n \times n} \\
-M^{-1} \cdot K & -M^{-1} \cdot C
\end{array}\right] \\
W & =\left[\begin{array}{c}
0 \\
M^{-1} \cdot F
\end{array}\right] \\
B & =\left[\begin{array}{c}
0 \\
M^{-1} \cdot E
\end{array}\right],
\end{aligned}
$$

where $A, W$, and $E$ are state matrices. $I$ is a unit matrix. According to the rank criterion, the system (see (7)) is observable and controllable. Therefore, structural vibration can be effectively suppressed by designing $U$ properly.

It is considered that the actuator would be only installed at isolation layer. For state equation (6), the maximum displacement value of the isolation layer, which is assumed, is $s$. Using $\underline{v}=x-\operatorname{sgn}(x) \cdot s$, obviously,

$$
\ddot{x}=\ddot{v} .
$$


From the above, the following can be achieved:

$$
(m \underline{\ddot{v}}+c \underline{\dot{v}}+k \underline{v})=-m \ddot{x}_{g}+u \text {. }
$$

Set $z=\left(z_{1}, z_{2}\right)^{T}=(\underline{v}, \underline{\dot{v}})^{T}$, and choose $u=c \underline{\dot{v}}+k \underline{v}+m \ddot{x}_{g}+$ $m v_{1}$; the following is achieved:

$$
\dot{z}=\left[\begin{array}{ll}
0 & 1 \\
0 & 0
\end{array}\right] \cdot z+\left[\begin{array}{l}
0 \\
1
\end{array}\right] \cdot v_{1} .
$$

The system is rewritten as follows:

$$
\begin{aligned}
& \dot{z}_{1}=z_{2} \\
& \dot{z}_{2}=v_{1} .
\end{aligned}
$$

Let

$$
v_{1}=-k_{1} \cdot \operatorname{sgn}\left(z_{1}\right) \cdot\left|z_{1}\right|^{\alpha_{1}}-k_{2} \cdot \operatorname{sgn}\left(z_{2}\right) \cdot\left|z_{2}\right|^{\alpha_{2}},
$$

where $k_{1}>0, k_{2}>0,0<\alpha_{1}<1, \alpha_{2}=2 \alpha_{1} /\left(1+\alpha_{1}\right), i=$ $1,2, \ldots, n$.

3.2. Stable Analysis of Nonsmooth Algorithms. Lyapunov function of the subsystem is selected as

$$
F_{1}\left(z_{1}, z_{2}\right)=\frac{k_{1}}{1+\alpha_{1}}\left|z_{1}\right|^{1+\alpha_{1}}+\frac{1}{2} z_{2}^{2}
$$

The derivation of (14) is as follows:

$$
\dot{F}_{1}\left(z_{1}, z_{2}\right)=-k_{2}\left|z_{2}\right|^{1+\alpha_{2}} \text {. }
$$

Obviously, $\dot{F}_{1}\left(z_{1}, z_{2}\right)$ is half negative. Therefore, the system is stable. The following set is considered as

$$
V_{1}=\left\{\left(z_{1}, z_{2}\right): \dot{F}\left(z_{1}, z_{2}\right)\right\} .
$$

By (11), the following is achieved:

$$
V_{1}=\left\{\left(z_{1}, z_{2}\right): z_{2}=0\right\} \text {. }
$$

Let $z_{2}(t)=0$; then we have $\dot{z}_{1}(t)=\dot{z}_{2}(t)=0$. When we substitute it into the closed-loop equations of system, it could be achieved; it is $z_{1}(t)=0$. Hence, the contained largest invariant set of the set $V_{1}$ is as follows:

$$
\left(\begin{array}{ll}
z_{1} & z_{2}
\end{array}\right)=\left(\begin{array}{ll}
0 & 0
\end{array}\right) \text {. }
$$

According to the invariance principle, in the equilibrium point $\left(\begin{array}{ll}z_{1} & z_{2}\end{array}\right)=\left(\begin{array}{ll}0 & 0\end{array}\right)$, the system is globally asymptotically stable and $f\left(z_{1}, z_{2}\right)=\left[f_{1}, f_{2}\right]^{T}=\left[\dot{z}_{1}, \dot{z}_{2}\right]^{T}$ is set. According to the theorem of homogeneous system [13], if the homogeneity of $f$ is satisfied, the formula is established as follows:

$$
\begin{aligned}
& f\left(\varepsilon^{\rho_{1}} z_{1}, \varepsilon^{\rho_{2}} z_{2}\right) \\
& =\left[\begin{array}{c}
\varepsilon^{\rho_{2}} y_{2 i} \\
-k_{1} \varepsilon^{\rho_{1} \alpha_{1}} \operatorname{sgn}\left(z_{1}\right)\left|z_{1}\right|^{\alpha_{1}}-k_{2} \varepsilon^{\rho_{2} \alpha_{2}} \operatorname{sgn}\left(z_{2}\right)\left|z_{2}\right|^{\alpha_{2}}
\end{array}\right] \\
& =\left[\begin{array}{cc}
\varepsilon^{\rho_{1}+l} & 0 \\
0 & \varepsilon^{\rho_{2}+l}
\end{array}\right]\left[\begin{array}{c}
y_{2 i} \\
-k_{1} \operatorname{sgn}\left(z_{1}\right)\left|z_{1}\right|^{\alpha_{1}}-k_{2} \operatorname{sgn}\left(z_{2}\right)\left|z_{2}\right|^{\alpha_{2}}
\end{array}\right] \\
& =\left[\begin{array}{c}
\varepsilon^{\rho_{1}+l} f_{1}\left(z_{1}, z_{2}\right) \\
\varepsilon^{\rho_{2}+l} f_{2}\left(z_{1}, y_{2}\right)
\end{array}\right] .
\end{aligned}
$$

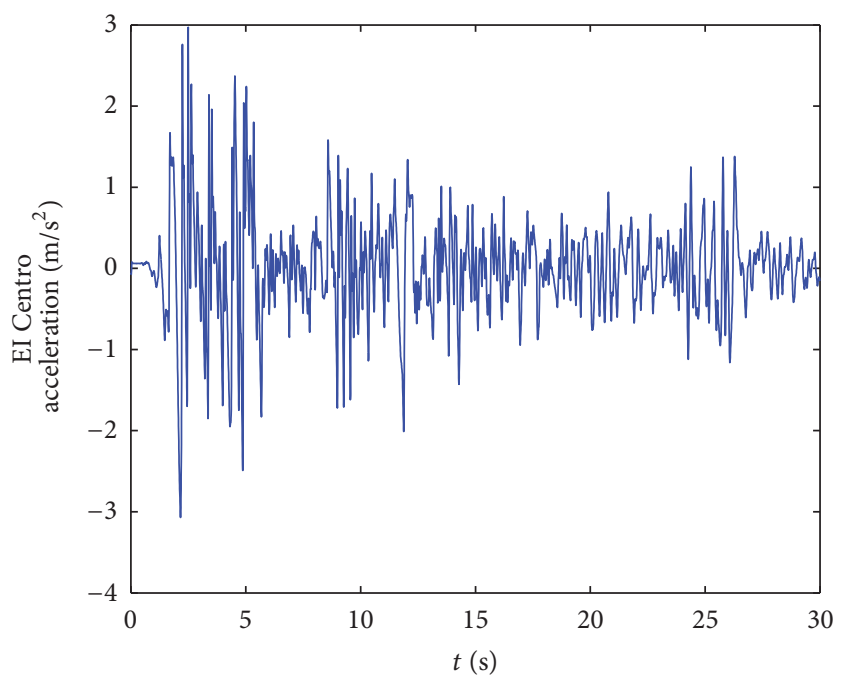

FIGURE 1: Seismic wave.

It can be got as

$$
\begin{aligned}
& \rho_{1}+l=\rho_{2} \\
& \rho_{2}+l=\rho_{1} \cdot \alpha_{1}=\rho_{2} \cdot \alpha_{2} .
\end{aligned}
$$

Let $\rho_{1}=1, \rho_{2}=\left(1+\alpha_{1}\right) / 2$. Equation (6) is established and $f\left(z_{1}, z_{2}\right)$ is satisfied with homogeneity. It means that the system is a homogeneous system. The degree of homogeneity in the system is $l=\left(\alpha_{1}-1\right) / 2<0$ which means negative degree of the homogeneity. Thus, system (9) is globally finitetime stable.

\section{Numerical Results of Examples}

In order to illustrate the effectiveness of the semiactive nonsmooth control algorithm, two three-layer base isolation structure models are analyzed with different parameters.

Model parameters of example 1 are as follows.

The masses of superstructure are $m_{1}=m_{2}=m_{3}=$ $9.167 \times 10^{5} \mathrm{~kg}$. The stiffness of superstructure is $k_{1}=k_{2}=$ $k_{3}=1.54 \times 10^{6} \mathrm{kN} / \mathrm{m}$. The damping coefficients of the superstructure are $c_{1}=c_{2}=c_{3}=4.813 \times 10^{7} \mathrm{kNs} / \mathrm{m}$. The mass of the isolation layer is $m_{b}=8.74 \times 10^{5} \mathrm{~kg}$. The stiffness of the isolation layer is $k_{b}=0.056 \times 10^{9} \mathrm{kN} / \mathrm{m}$. The damping coefficient of the isolation layer is $c_{b}=0.832 \times 10^{7} \mathrm{kNs} / \mathrm{m}$. The requirement of horizontal displacement is $20 \mathrm{~mm}$.

El Centro Seismic wave, the unknown disturbance, is estimated by deep neural network. The result is shown in Figure 1 and peek value of acceleration is $3 \mathrm{~m} / \mathrm{s}^{2}$. According to the parameters of the aforementioned model, the dynamic responses are simulated in two ways of semiactive nonsmooth control and passive control. The displacements, velocities, and acceleration and control forces of the seismic isolation are shown in Figures 2-4. In order to further analyze the effect of semiactive nonsmooth control and passive control, the maximum displacement $x_{b \max }$ and the maximum acceleration $a_{b \max }$ in the seismic isolation are made statistics. The results are shown in Table 1. 


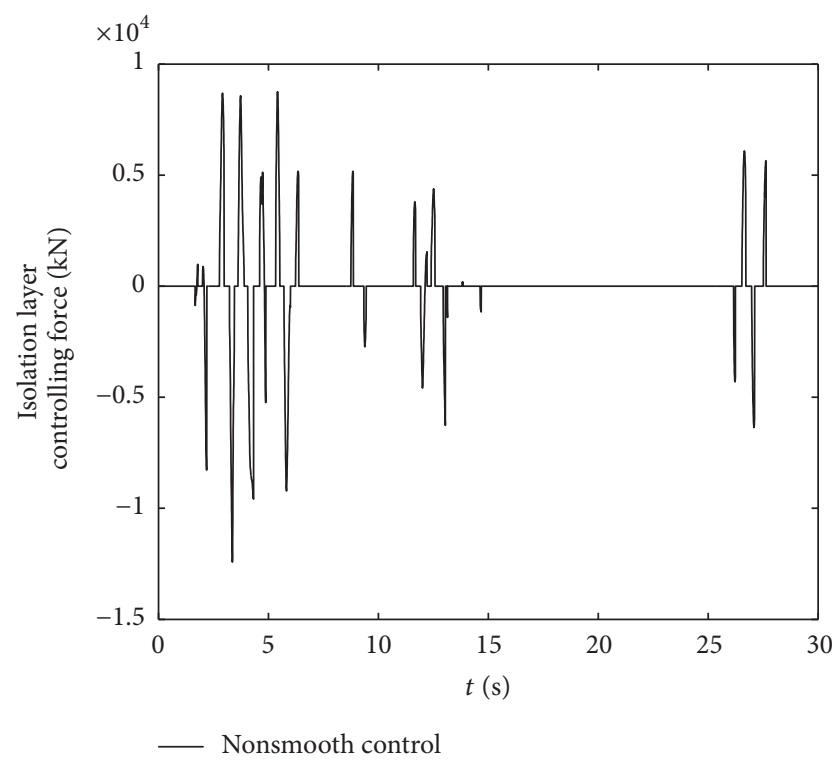

FIGURE 2: Control force response of isolation layer.

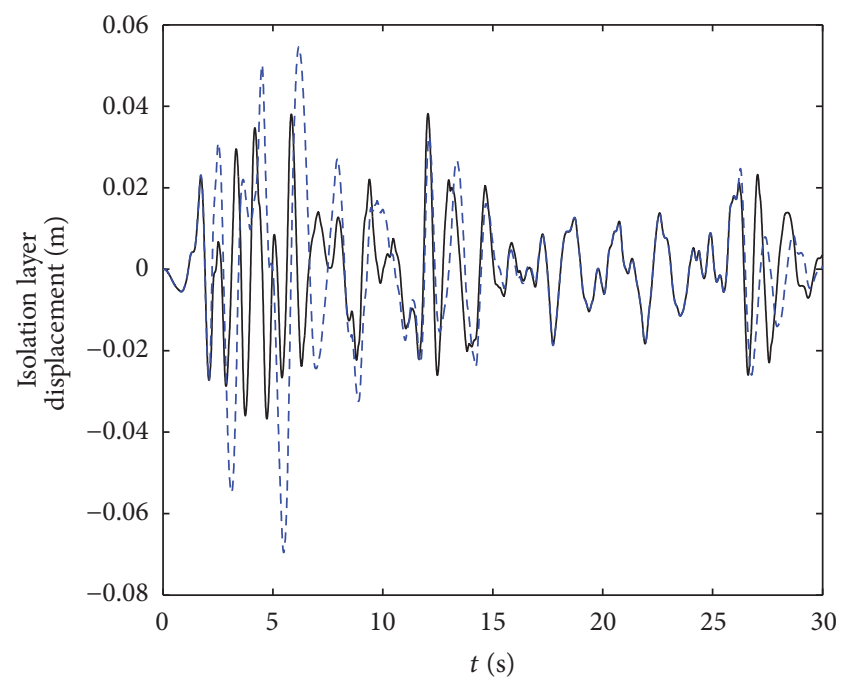

— Nonsmooth control - _- Passive control

FIGURE 3: Displacement response of isolation layer.

TABLE 1: Comparison of peak data.

\begin{tabular}{lcc}
\hline Control strategy & $x_{b \max }(\mathrm{m})$ & $a_{b \max }\left(\mathrm{m} / \mathrm{s}^{2}\right)$ \\
\hline Isolation layer & & \\
Passive control & 0.070 & 3.300 \\
Nonsmooth semiactive control & 0.038 & 1.171 \\
\hline
\end{tabular}

As shown in Figures 1-5, in the range of the external control force, the velocity of isolation layer with semiactive nonsmooth control is a little larger than passive control. But in the terms of cutting down the displacements and acceleration of isolation layer, semiactive nonsmooth control is superior to passive control. As is shown in Table 1, the peak

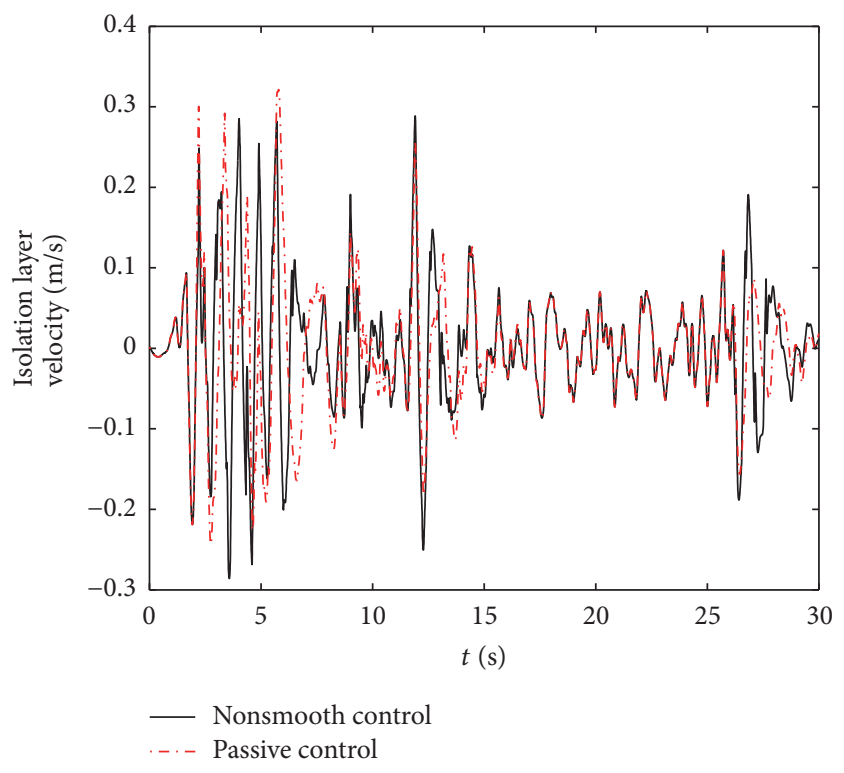

FIGURE 4: Velocity response of isolation layer.

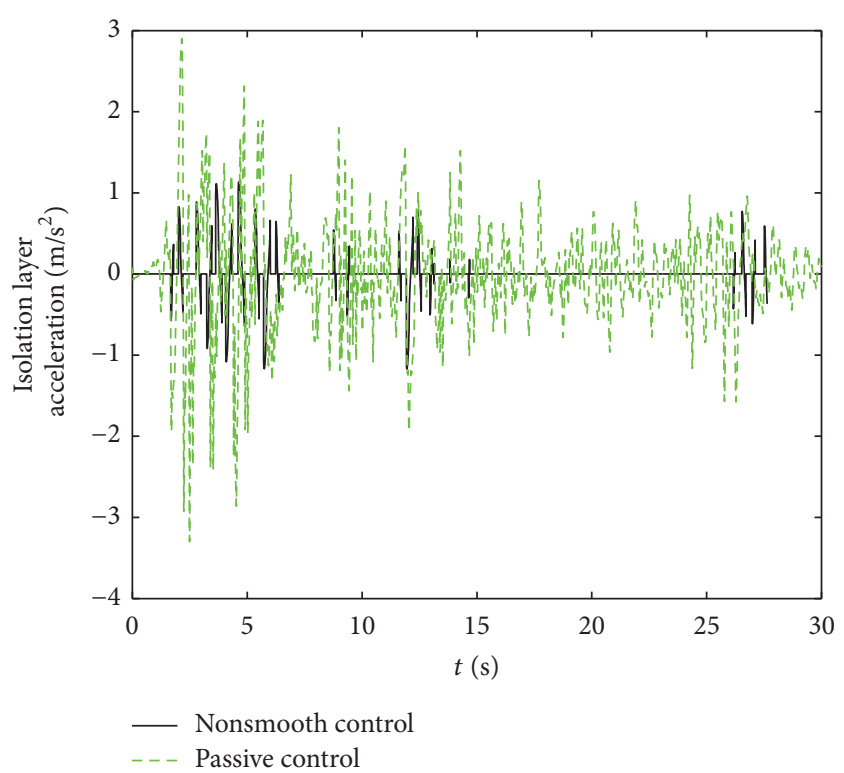

Figure 5: Acceleration response of isolation layer.

acceleration of isolation layer with semiactive nonsmooth control is down to $35 \%$. Compared with passive control, the peak displacement of isolation layer is decreased by $46 \%$. In the isolation layer, the horizontal distortion is reduced drastically. Hence, control effect is obvious.

Model parameters of example 2 are as follows.

The masses of superstructure are $m_{1}=53200 \mathrm{~kg}, m_{2}=$ $61300 \mathrm{~kg}$, and $m_{3}=54500 \mathrm{~kg}$. The stiffness of superstructure is $k_{1}=115.3 \times 10^{3} \mathrm{kN} / \mathrm{m}, k_{2}=115.3 \times 10^{3} \mathrm{kN} / \mathrm{m}$, and $k_{3}=$ $74.9 \times 10^{3} \mathrm{kN} / \mathrm{m}$. The damping coefficients of superstructure are $c_{1}=171.5 \mathrm{kNs} / \mathrm{m}, c_{2}=171.5 \mathrm{kNs} / \mathrm{m}$, and $c_{3}=$ $111.4 \mathrm{kNs} / \mathrm{m}$. The mass of isolation layer is $m_{b}=41 \times 10^{3} \mathrm{~kg}$. The stiffness of isolation layer is $k_{b}=1.5 \times 10^{3} \mathrm{kN} / \mathrm{m}$. The 


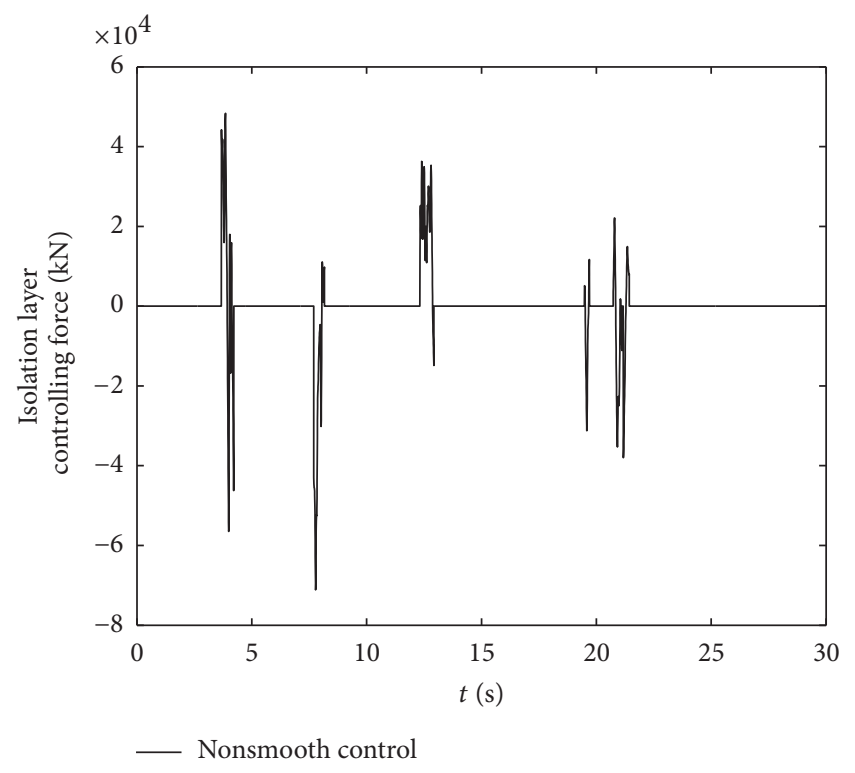

FIGURE 6: Control force response of isolation layer.

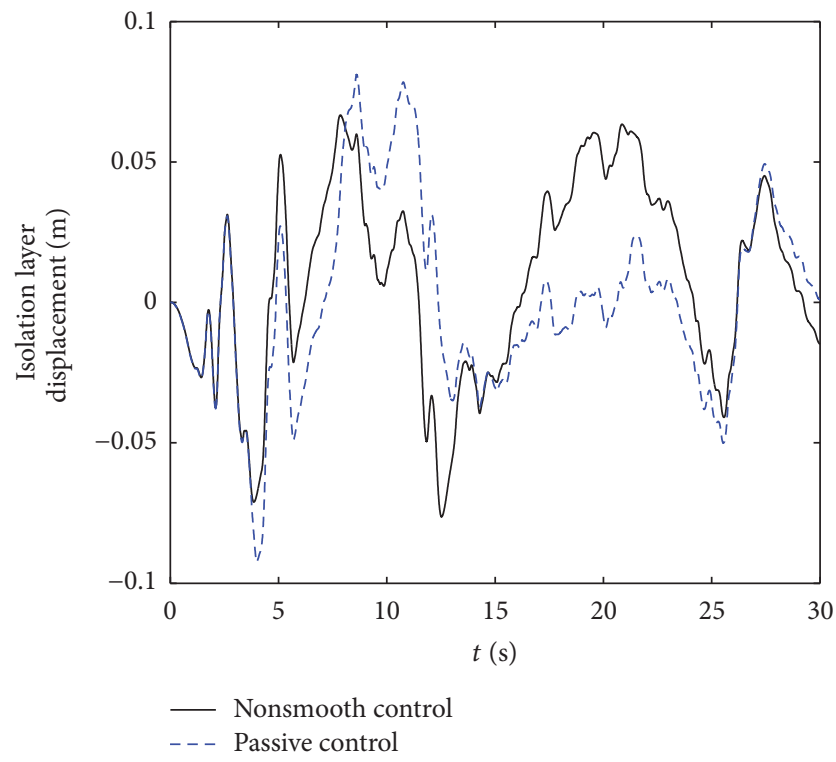

Figure 7: Displacement response of isolation layer.

damping coefficient of the isolation layer is $c_{b}=53.5 \mathrm{kNs} / \mathrm{m}$. The requirement of horizontal displacement is $60 \mathrm{~mm}$.

Example 2 inputs seismic wave El Centro and is simulated and analyzed. The requirement of horizontal displacement is a criterion. And the proposed model is in the two conditions, respectively, semiactive nonsmooth control and passive control. The contrastive curves, which include displacements, velocities, and acceleration, are shown in Figures 7-9. The peak displacement $x_{b \max }$ and the peak acceleration $a_{b \max }$ in the isolation layer of the proposed model are counted. They are showed in Table 2.

As is shown in time-history curves in Figures 6-9, the conclusion can be drawn similarly. The displacement and peak displacement in the isolation layer are obviously

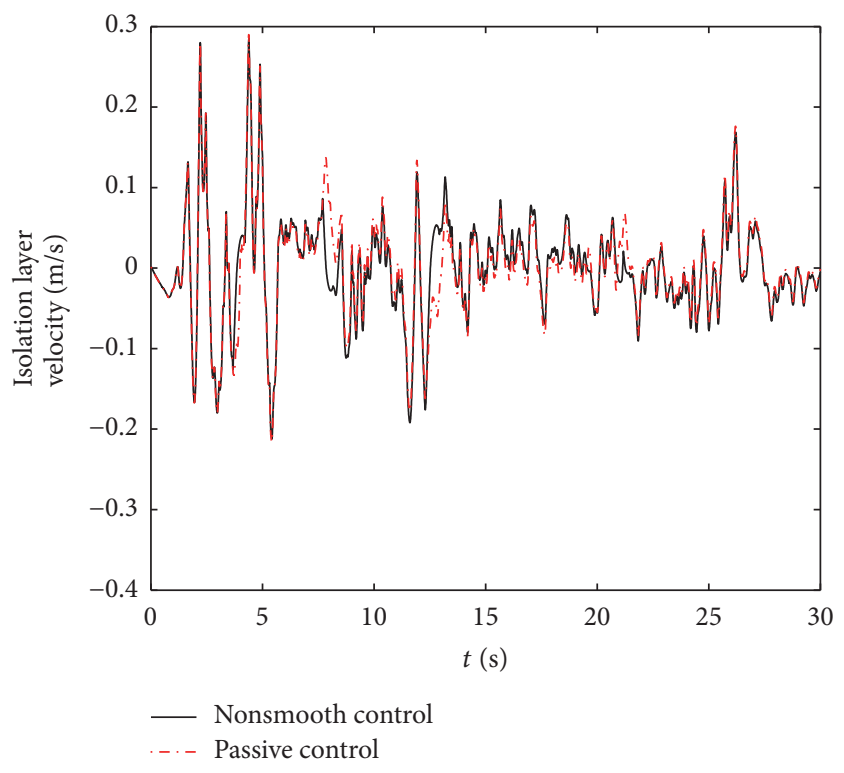

FIGURE 8: Velocity response of isolation layer.

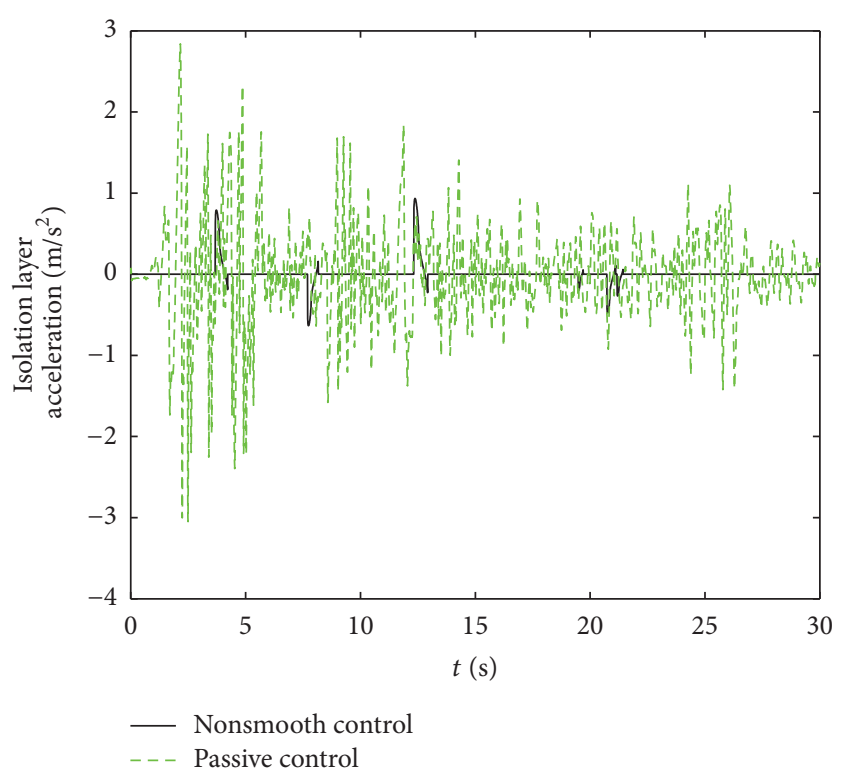

FIGURE 9: Acceleration response of isolation layer.

TABLE 2: Comparison of peak data.

\begin{tabular}{lcc}
\hline Control strategy & $x_{b \max }(\mathrm{m})$ & $a_{b \max }\left(\mathrm{m} / \mathrm{s}^{2}\right)$ \\
\hline Isolation layer & & \\
$\quad$ Passive control & 0.0925 & 3.0703 \\
Nonsmooth semiactive control & 0.0764 & 0.9333 \\
\hline
\end{tabular}

decreased in the semiactive nonsmooth control system. As is shown in Table 2, the acceleration of the model in isolation layer is also declined. It means the peak acceleration is controlled. Compared with the passive control system, the peak displacement of the isolation layer decreases by $21 \%$ with semiactive nonsmooth control system. The horizontal 
peak displacement in isolation layer is declined drastically. It shows that semiactive nonsmooth control algorithm is able to improve performance of passive isolation structure to prevent isolation layer from too much deformation.

From the simulated analyses of two models with different parameters, the semiactive nonsmooth control algorithm can cut down dynamic response more effective than passive control. The displacement of the structure is controlled to allowable range as much as possible. The disadvantage of excessive displacement in the isolation layer is solved effectively, so the effect of designed control is remarkable.

\section{Conclusions}

Semiactive nonsmooth control with Deep Learning is more effective than passive control. By using deep neural network, the designed control method not only considers the unknown term but also cuts down the structural peak response that is caused by earthquake. The analysis of seismic response in the controlled structure shows that the proposed algorithm improves the performance of isolated structure and reduces the seismic response in the isolation layer and superstructure. The designed control method prevents building structure from oversize deformation that causes structural failure. Thus, the designed control method is high-effect. Semiactive nonsmooth control algorithm has great robustness and can restrain the influence of seismic wave effectively. Thus, the designed nonsmooth control algorithm with Deep Learning is more intellectualized, feasible, and effective. The proposed control method could be theoretical basis for practical building structural engineering application.

\section{Conflicts of Interest}

The authors declare that there are no conflicts of interest regarding the publication of this paper.

\section{Acknowledgments}

This work is supported by National Natural Science Foundation (NNSF) of China (under Grant no. 51478132) and Guangzhou City College Scientific Research Project (under Grant no. 120163017).

\section{References}

[1] S. Etedali, M. R. Sohrabi, and S. Tavakoli, "Optimal PD/PID control of smart base isolated buildings equipped with piezoelectric friction dampers," Earthquake Engineering and Engineering Vibration, vol. 12, no. 1, pp. 39-54, 2013.

[2] J. Yao, "Concept of structural control," Journal of the Structural Division, vol. 98, no. 7, pp. 1567-1574, 1972.

[3] H. Hu, D. Guo, and J. Weng, "Recent advances in semi-active control of vibration," Journal of Vibration, Measurement \& Diagnosis, vol. 4, pp. 235-244, 2001.

[4] F. Zhou, Engineering Structure Vibration Control, Seismological Press, Beijing, China, 1997.

[5] X.-M. Li and Y.-F. Du, "Curved girder bridges' control based on sequential optimal control algorithm under two-directional horizontal earthquake," Journal of Vibration \& Shock, vol. 34, no. 10, pp. 6-33, 2015.

[6] K. K. F. Wong and R. Yang, "Predictive instantaneous optimal control of inelastic structures during earthquakes," Earthquake Engineering \& Structural Dynamics, vol. 32, no. 14, pp. 21792195, 2010.

[7] J. Ou, Structural Vibration Control-Active, Semi- Active and Intelligent Control, Science Press, Beijing, China, 2003.

[8] S. Tong and H. Tang, "Iterative learning instantaneous optimal control of discrete systems optimization of actuator positions," Applied Mathematics and Mechanics, vol. 37, no. 2, pp. 160-172, 2016.

[9] M. Battaini, F. Casciati, and L. Faravelli, "Fuzzy control of structural vibration. An active mass system driven by a fuzzy controller," Earthquake Engineering \& Structural Dynamics, vol. 27, no. 11, pp. 1267-1276, 1998.

[10] J. N. Yang, J. C. Wu, K. Kawashima, and S. Unjoh, "Hybrid control of seismic-excited bridge structures," Earthquake Engineering \& Structural Dynamics, vol. 24, no. 11, pp. 1437-1451, 1995.

[11] J. H. Wang, Z. Liu, C. Chen, and Y. Zhang, "Fuzzy adaptive compensation control of uncertain stochastic nonlinear systems with actuator failures and input hysteresis," IEEE Transactions on Cybernetics, vol. PP, no. 99, pp. 1-12, 2017.

[12] X. Lin and R. Zhang, " $\mathrm{H}_{\infty}$ control for stochastic systems with Poisson jumps," Journal of Systems Science and Complexity, vol. 24, no. 4, pp. 683-700, 2011.

[13] K.-M. Ma, "Design of continuous non-smooth attitude control laws for spacecraft," Journal of Astronautics, vol. 33, no. 6, pp. 713-719, 2012.

[14] Q. Wang, J. Wang, K. Ma et al., "Nonsmooth control algorithm for structural vibration control and its applications," Earth Engineering and Engineering Dynamics, vol. 36, no. 5, pp. 207214, 2016

[15] J. Wang, Q. Wang, and K. Ma, "Non-smooth controller design for permanent magnet synchronous motors," Computer Simulation, vol. 33, no. 3, pp. 227-230, 2016.

[16] K.-M. Ma, "Non-smooth design and implementation of highprecision guidance laws," Journal of Ballistics, vol. 25, no. 2, pp. $1-5,2013$.

[17] K. Ma, "Design of continuous non-smooth attitude control laws for spacecraft," Journal of Ballistics, vol. 33, no. 6, pp. 713-719, 2016.

[18] H. Liu, S. Ding, S. Li et al., "Finite-time control of PSPM position servo system," Electric Machines and Control, vol. 13, no. 3, pp. 424-430, 2009.

[19] Y. Bengio, É. ThibodeauLaufer, G. Alain et al., "Deep generative stochastic networks trainable by backprop," Computer Science, vol. 2, pp. 226-234, 2014.

[20] L. Deng, M. Seltzer, D. Yu et al., "Binary coding of speech spectrograms using a deep auto-encoder," in Proceedings of the Conference of the International Speech Communication Association (INTERSPEECH '10), pp. 1692-1695, Makuhari, Chiba, Japan, September 2010.

[21] G. E. Hinton, A. Krizhevsky, and S. D. Wang, "Transforming auto-encoders," in Artificial Neural Networks and Machine Learning, vol. 6791 of Lecture Notes in Computer Science, pp. 4451, 2011.

[22] P. Vincent, "A connection between score matching and denoising autoencoders," Neural Computation, vol. 23, no. 7, pp. 16611674, 2011. 
[23] O. Vinyals, Y. Jia, L. Deng et al., "Learning with recursive perceptual representations," Advances in Neural Information Processing Systems, pp. 2834-2842, 2012.

[24] C. Yang, X. Wang, L. Cheng, and H. Ma, "Neural-learningbased telerobot control with guaranteed performance," IEEE Transactions on Cybernetics, vol. PP, no. 99, pp. 1-12, 2017.

[25] C. Yang, Z. Li, and J. Li, “Trajectory planning and optimized adaptive control for a class of wheeled inverted pendulum vehicle models," IEEE Transactions on Cybernetics, vol. 43, no. 1, pp. 24-36, 2013.

[26] H. Xiao, Z. Li, C. Yang et al., "Robust stabilization of a wheeled mobile robot using model predictive control based on neurodynamics optimization," IEEE Transactions on Industrial Electronics, vol. 64, no. 1, pp. 505-516, 2017.

[27] C. Yang, X. Wang, Z. Li et al., "Teleoperation control based on combination of wave variable and neural networks," IEEE Transactions on Systems Man \& Cybernetics Systems, vol. PP, no. 99, pp. 1-12, 2017.

[28] C. Yang, J. Luo, Y. Pan et al., "Personalized variable gain control with tremor attenuation for robot teleoperation," IEEE Transactions on Systems Man \& Cybernetics Systems, vol. PP, no. 99, pp. 1-12, 2017.

[29] X. Z. Meng, S. N. Zhao, T. Feng, and T. H. Zhang, "Dynamics of a novel nonlinear stochastic SIS epidemic model with double epidemic hypothesis," Journal of Mathematical Analysis and Applications, vol. 433, no. 1, pp. 227-242, 2016.

[30] J. Tang and Z. Lium, Isolated Structure Design, Huazhong University of Science and Technology Press, Wuhang, China, 1993.

[31] J. M. Kelly, Earthquake-Resistant Design with Rubber, Springer, London, England, 1993.

[32] G. Li, J. Li, and X. Su, Seismic Design of Building Structures, China Building Industry Press, 2nd edition, 2007.

[33] M. Liu, X. Tian, D. Wang et al., "Research and application of building structure base isolation technology," Technology for Earthquake Disaster Prevention, vol. 1, no. 1, pp. 31-38, 2006.

[34] Z. Zhao, X. Wang, C. Zhang, Z. Liu, and J. Yang, "Neural network based boundary control of a vibrating string system with input deadzone," Neurocomputing, In Press.

[35] F. Wang, B. Chen, C. Lin, J. Zhang, and X. Meng, "Adaptive neural network finite-time output feedback control of quantized nonlinear systems," IEEE Transactions on Cybernetics, vol. PP, no. 99, pp. 1-10, 2017.

[36] H. Cheng and T. Zhang, "A new predator-prey model with a profitless delay of digestion and impulsive perturbation on the prey," Applied Mathematics and Computation, vol. 217, no. 22, pp. 9198-9208, 2011.

[37] X. Dong, Z. Bai, and S. Zhang, "Positive solutions to boundary value problems of $p$-Laplacian with fractional derivative," Boundary Value Problems, vol. 2017, article 5, 2017.

[38] Z. Bai, S. Zhang, S. Sun, and C. Yin, "Monotone iterative method for fractional differential equations," Electronic Journal of Differential Equations, vol. PP, no. 99, pp. 1-12, 2016. 


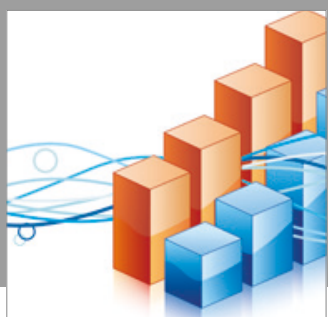

Advances in

Operations Research

vatersals

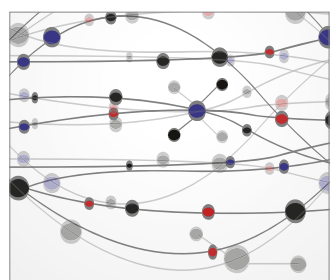

\section{The Scientific} World Journal
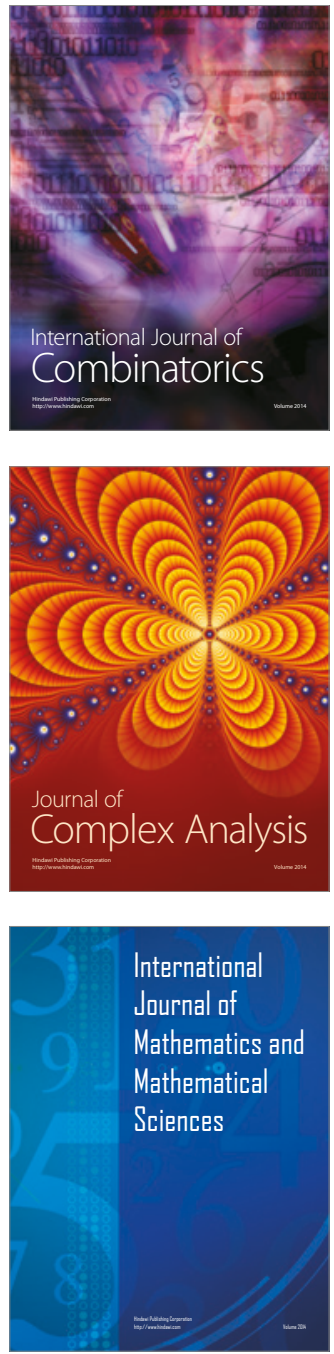
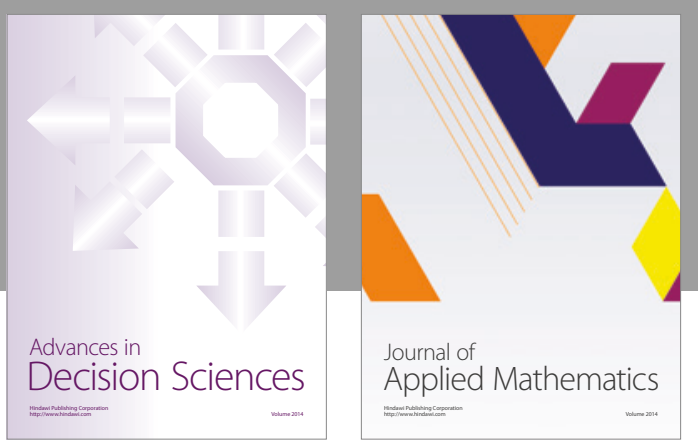

Algebra

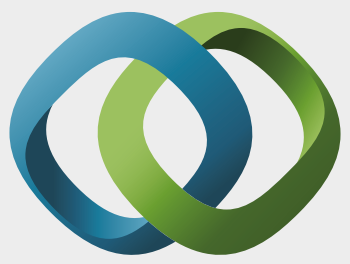

\section{Hindawi}

Submit your manuscripts at

https://www.hindawi.com
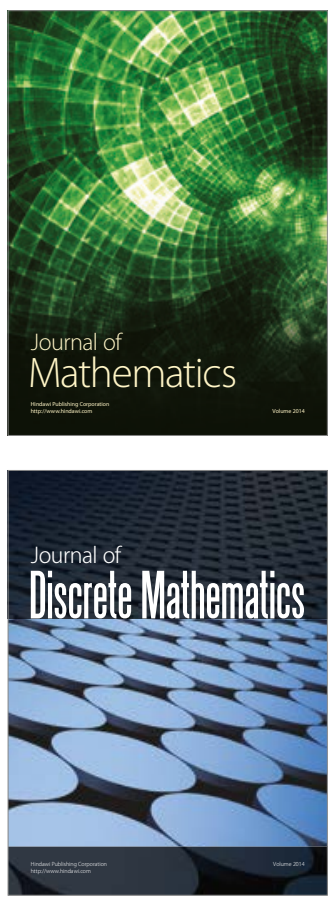

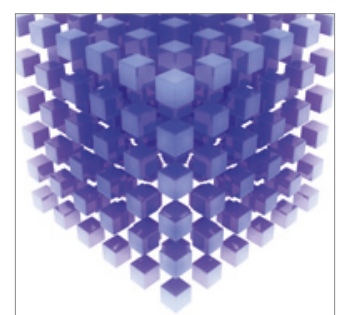

Mathematical Problems in Engineering
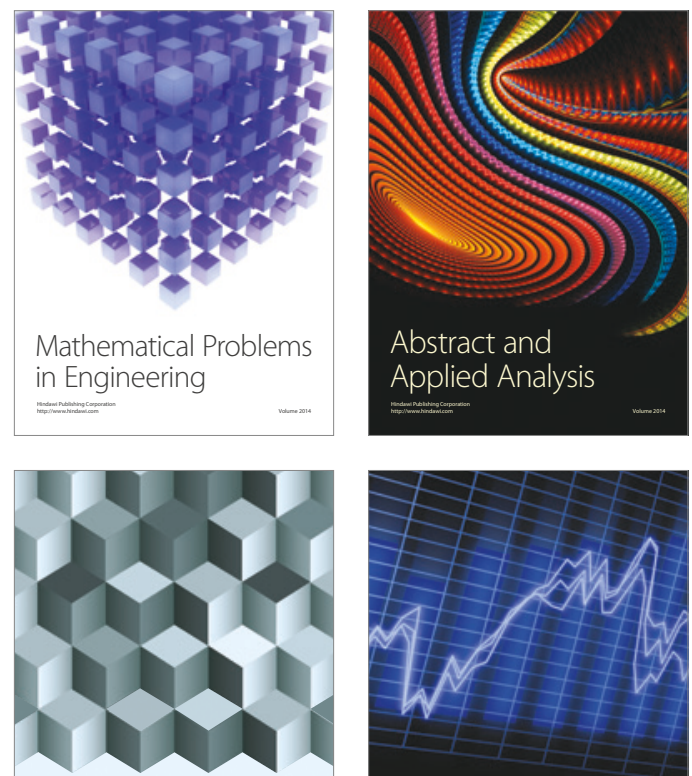

Journal of

Function Spaces

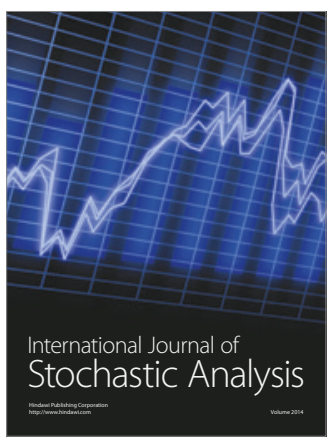

Probability and Statistics
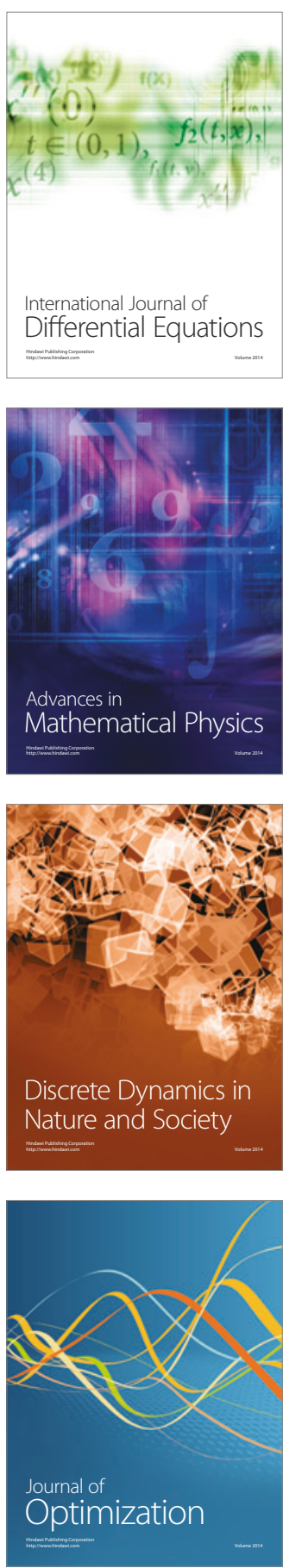